\title{
Tracheostomy Tube Displacement: An Update on Emergency Airway Management
}

\author{
Rajkumar Rajendram ${ }^{1,2}$, Muhammad Faisal Khan ${ }^{3}$, Alex Joseph ${ }^{4}$ \\ ${ }^{1}$ Department of Medicine, King Abdulaziz Medical City, Ministry of National Guard Health Affairs, Riyadh, Saudi Arabia, ${ }^{2}$ Department of Anaesthesia and Intensive \\ Care, Stoke Mandeville Hospital, Stoke Mandeville, Buckinghamshire, UK, ${ }^{3}$ Department of Anaesthesiology, Aga Khan University, Karachi, Pakistan, ${ }^{4}$ Department of \\ Anaesthesia, Changi General Hospital, Singapore
}

\section{Abstract}

The formation of tracheal stomas and insertion of tracheostomy tubes is increasing in frequency. Although tracheostomy tube displacement is uncommon, the associated mortality is high. This is because rapid intervention is required, management is often difficult and even specialists in airway management often have limited experience with tracheostomized patients. Anyone caring for a patient with a tracheostomy should be aware of the clinical presentation of tracheostomy tube displacement and be able to manage it rapidly. This review describes the application of fundamental principles of airway management to tracheostomy tube displacement to reinforce its similarity to most other airway emergencies. The first and most important question is: Does the airway need to be re-secured? If so can the tracheostomy be reinserted easily? Assessment of the patient's upper airway must determine whether translaryngeal oxygenation and endotracheal intubation are possible. Information about the other factors that influence management can be obtained from the medical records about the formation of the tracheal stoma. Even airway specialists find it challenging to acquire and process this information to formulate an appropriate management plan, off the cuff, in the heat of the moment. Hence, multidisciplinary guidelines for the management of tracheostomy and laryngectomy airway emergencies recommend that this information be displayed on posters at the bedside. This poster should also be accompanied by a written plan for emergency orotracheal intubation or tracheostomy replacement in the event of an airway emergency. All the equipment required to execute these plans should be immediately available at the bedside.

Keywords: Airway, dislodgement, displacement, tracheostomy

\section{INTRODUCTION}

Tracheostomy tube displacement is rare but can be fatal. A recent study reported that the incidence was $<1 / 1000$ tracheostomy tube days. ${ }^{[1]}$ However, this airway emergency was the most common cause of major morbidity and mortality from airway management in Intensive Care Unit (ICU) in the United Kingdom during the national audit of the major complications of airway management conducted by the Royal College of Anaesthetists (The $4^{\text {th }}$ National Audit Project; NAP4). ${ }^{[2]}$ Half of the 14 patients whose tracheostomy tubes displaced during NAP4 died ${ }^{[2]}$ Hence, while the incidence of displacement is very low, the associated risk of mortality is very high. ${ }^{[1-4]}$ It is therefore vital to rapidly detect displacement of a tracheostomy tube and urgently obtain help from a senior specialist in airway management who has experience with tracheostomized patients. However, even experienced airway specialists can feel out of their depth in this unfamiliar situation.

\begin{tabular}{|l|l|}
\hline \multicolumn{2}{|c|}{ Access this article online } \\
\hline Quick Response Code: & Website: \\
& www.ijrconline.org \\
\cline { 2 - 2 } & \\
\end{tabular}

This review describes the application of fundamental principles of the management to tracheostomy tube displacement. It aims to reinforce the similarity of the management of this situation to that of most other airway emergencies.

\section{Simplicity of Management of Tracheostomy DisPLACEMENT}

In a patient in whom tracheostomy tube displacement is suspected the airway mantra "if in doubt, take it out!" must always be remembered. Hence, the tracheostomy tube should usually be removed if not displaced completely. In some

Address for correspondence: Dr. Rajkumar Rajendram, Department of Anaesthesia, Stoke Mandeville Hospital, Stoke Mandeville, Buckinghamshire, UK E-mail: rajkumarrajendram@doctors.org.uk

This is an open access article distributed under the terms of the Creative Commons Attribution-NonCommercial-ShareAlike 3.0 License, which allows others to remix, tweak, and build upon the work non-commercially, as long as the author is credited and the new creations are licensed under the identical terms.

For reprints contact: reprints@medknow.com

How to cite this article: Rajendram R, Khan MF, Joseph A. Tracheostomy tube displacement: An update on emergency airway management. Indian J Respir Care 2017;6:800-6. 
Rajendram, et al:: Tracheostomy tube displacement

situations, a decannulation trial may then be considered but, in most cases, the airway should be re-secured urgently. This can often be achieved by reinsertion of the tracheostomy tube.

If the tracheostomy tube cannot be replaced management can be extremely challenging. When translaryngeal oxygenation and endotracheal intubation ARE possible; attempting to maintain and re-secure the airway from above can be considered instead. In this situation, the approach to management should be familiar to all airway specialists. However, it is important to ensure that the tracheostomy tract and tracheal stoma are covered while attempts are made to maintain and re-secure the airway from above using a facemask, supraglottic airway or oro-tracheal endotracheal intubation.

If translaryngeal oxygenation and endotracheal intubation are NOT possible (e.g., postlaryngectomy) management will be perceived as being extremely difficult. This misconception arises because this potentially life-threatening situation is very unfamiliar, even to very experienced airway specialists. The fundamental principles are actually very similar to the management of most airway emergencies. However, the upper airway may appear relatively normal. Hence, the real challenge is actually in determining that there are in fact no alternatives. The airway can only be maintained and re-secured via the trachea! Once this has been recognized, airway management is greatly simplified as the range of options is significantly reduced. Fruitless attempts to manage the airway from above can be avoided.

If immediate reinsertion of the tracheostomy is not straightforward, oxygen can be administered through a facemask, trachemask or supraglottic airway applied externally over the stoma. In most cases, a supraglottic airway with an inflatable cuff (e.g., laryngeal mask airway) can provide sufficient seal around the tracheal stoma to allow ventilation. If an appropriate tracheostomy tube is not available, an endotracheal tube may be inserted via the stoma. If everything else fails then emergency cricotyroidotomy or emergency tracheostomy are required. Although the range of management options is limited, great care and attention to detail are still required.

There are many potential pitfalls in the management of tracheotomy tube displacement. For example, blind attempts to recannulate the stoma can create a false passage in the pretracheal space and or cause significant hemorrhage. Attempts to ventilate via a tube displaced into the pretracheal space will generate subcutaneous emphysema. This further distorts already unfamiliar anatomy and is therefore disastrous.

Those caring for patients with tracheostomies must be aware of the clinical presentation of a displaced tracheostomy tube. They must also be prepared and able to manage it rapidly. The preceding paragraphs highlight the similarity of the acute management of tracheostomy tube displacement to that of most airway emergencies. They also underscore the fundamental importance of determining whether translaryngeal oxygenation and endotracheal intubation are possible.

The rest of this review, therefore, focuses on the important factors which influence acute management and provides a guide to the formulation of emergency airway plans for this high-risk cohort.

\section{Factors That Influence the Management of Tracheostomy-related Emergencies}

After detecting a displaced tracheostomy tube, the first and most important question to answer is:

Does the airway need to be re-secured (immediately) or is a tracheostomy decannulation trial appropriate?

To answer this question, an initial rapid clinical assessment of the situation must determine:

1. If the tracheostomy tube is partially or completely displaced

2. If the patient has any respiratory distress

3. If the patient has respiratory distress, can this be managed without re-securing the airway?

If the airway must be re-secured immediately, then the whole team managing the emergency must be aware of the anatomy of the patient's upper airway, tracheostomy tract and tracheal stoma as well as the characteristics of the tracheostomy tube that was in situ.

The characteristics of tracheostomy tubes that affect the management of airway-related emergencies are highlighted in Table 1. The important information about the patient's upper airway and formation of the tracheostomy tract and tracheal stoma are listed in Tables 2 and 3, respectively. The answers to the questions in Tables 1-3 define the best management strategies for any individual patient who experiences a tracheostomy-related emergency complication. Perhaps

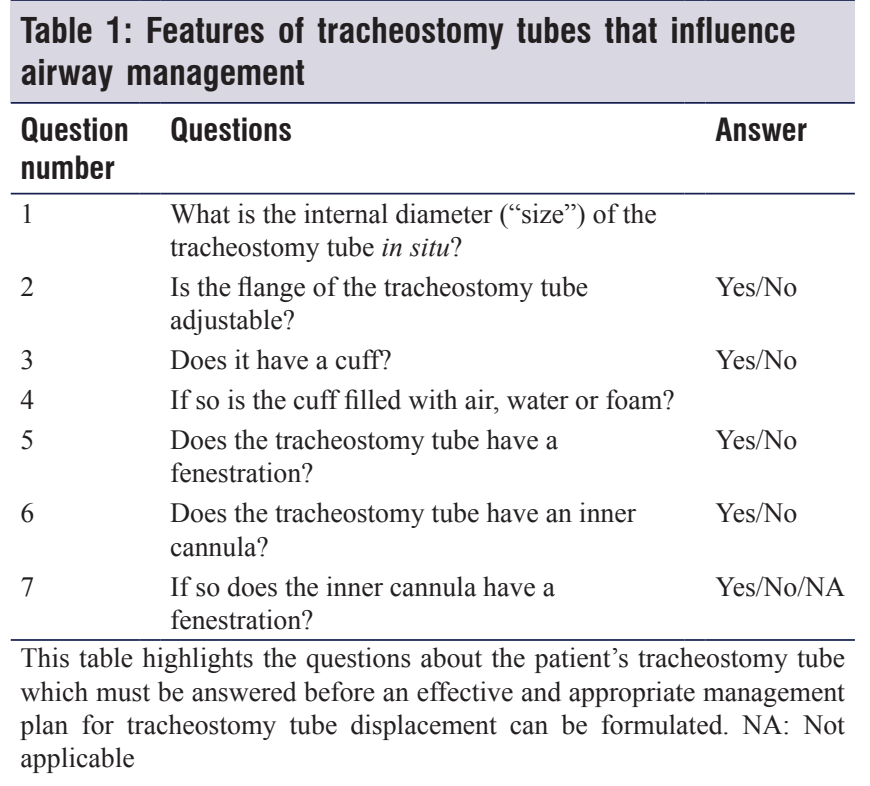


Rajendram, et al:: Tracheostomy tube displacement

\begin{tabular}{|c|c|c|}
\hline $\begin{array}{l}\text { Question } \\
\text { number }\end{array}$ & Questions & Answer \\
\hline \multirow[t]{7}{*}{1} & Is oro-tracheal intubation possible? & Yes/No \\
\hline & If yes & \\
\hline & What was the laryngoscopy grade? & 1234 \\
\hline & $\begin{array}{l}\text { Have there previously been any } \\
\text { difficulties with oro-tracheal intubation? } \\
\text { If yes }\end{array}$ & Yes/No \\
\hline & What is/was the cause of difficulty? & Yes/No \\
\hline & $\begin{array}{l}\text { How was the airway managed in that } \\
\text { situation? }\end{array}$ & \\
\hline & Has this issue been resolved? & Yes/No \\
\hline 2 & Has the patient had a laryngectomy? & Yes/No \\
\hline 3 & Is the upper airway patent? & Yes/No \\
\hline
\end{tabular}

\begin{tabular}{|c|c|c|}
\hline $\begin{array}{l}\text { Question } \\
\text { number }\end{array}$ & Question & Answer \\
\hline 1 & $\begin{array}{l}\text { What was the indication for formation of } \\
\text { the tracheal stoma? }\end{array}$ & \\
\hline 2 & $\begin{array}{l}\text { When was the tracheostomy tract and } \\
\text { tracheal stoma initially formed? }\end{array}$ & \\
\hline \multirow[t]{5}{*}{3} & $\begin{array}{l}\text { Was a percutaneous or surgical } \\
\text { technique used to form the tract and } \\
\text { stoma? }\end{array}$ & $\begin{array}{l}\text { Percutaneous } \\
\text { Surgical }\end{array}$ \\
\hline & If surgical & \\
\hline & Was the thyroid isthmus divided? & Yes/No \\
\hline & Was a Björk flap formed? & Yes/No \\
\hline & Are stay sutures present? & Yes/No \\
\hline \multirow[t]{5}{*}{4} & $\begin{array}{l}\text { Has the tracheostomy tract and tracheal } \\
\text { stoma ever been revised? }\end{array}$ & Yes/No \\
\hline & If so & \\
\hline & Why? & \\
\hline & When? & \\
\hline & How? & \\
\hline \multirow[t]{5}{*}{5} & $\begin{array}{l}\text { Has the patient had any complications } \\
\text { related to the tracheostomy? }\end{array}$ & Yes/No \\
\hline & If so & \\
\hline & What? & \\
\hline & When? & \\
\hline & How were they managed? & \\
\hline
\end{tabular}

This table highlights the questions about the patient's tracheostomy tract and stoma which must be answered before an effective and appropriate management plan for tracheostomy tube displacement can be formulated

more importantly, they also define what should not be tried (e.g., facemask ventilation and orotracheal intubation in a patient who has had a laryngectomy).

Tracheostomy tube displacement is a life-threatening emergency in which a delay of even a few seconds can make a difference to the outcome. Valuable time is wasted if the on-call team treating the patient out-of-hours have to search for this vital information themselves in this time-critical situation. Furthermore, even experienced airway specialists have difficulty processing this data to formulate and then execute an appropriate management plan, off the cuff, in the heat of the moment. This challenge is intensified by the chaotic environment that surrounds a patient with a displaced tracheostomy tube who is distressed.

All patients with a tracheostomy in situ must, therefore, have a written, patient-specific, emergency orotracheal intubation and tracheostomy recannulation plan prepared in advance. An example of an emergency management plan for tracheostomy tube displacement is provided in the NAP4 report. ${ }^{[2]}$

All the relevant stakeholders who may be expected to manage the patient in the event of an airway emergency should be able to execute this plan rapidly. This standard is easily achieved by the primary team who usually manage the patient "in hours." However, airway emergencies can occur at any time of the day or night. The current shift patterns of healthcare workers mean that it would be virtually impossible to communicate the specific emergency airway management plan for every patient with a tracheostomy to each "on call" team.

Hence, the British national tracheostomy safety project strongly recommends that this crucial information is made easily available at the bedside of any patient with a tracheostomy tube in situ. ${ }^{[5,6]} \mathrm{A}$ vital aspect of this safety campaign is the use of large, clearly visible, color-coded posters to differentiate those patients with a tracheostomy tube but have a patent upper airway from those who have had a laryngectomy. This distinction is of the utmost importance because it distinguishes those patients in whom orotracheal intubation is possible from those in whom the only possible route for oxygenation and ventilation is via the trachea.

It could be argued that displaying this sensitive medical information on a poster at the bedside constitutes a significant breach of patient confidentiality. However, in our experience, no one has refused to have the poster displayed at their bedside after receiving an explanation of the significant potential benefit to their safety and outcome in the event of a complication.

\section{The Relevance of the Technique Used to Form the TRACHEOSTOMY}

Techniques used to form tracheal stoma, and tracheostomy tracts can be classified as percutaneous or surgical. The technique used for formation of the tracheostomy affects the risk of complications such as tube displacement and the ease of recannulation.

For example, during a surgical tracheostomy, the thyroid isthmus may be either retracted or divided. An intact isthmus may descend, obstructing the stoma if the tube is displaced. The tracheal stoma is usually between the second and fourth cartilage rings of the trachea. The risk of displacement is increased if the tracheostomy is too low or off the midline. ${ }^{[7]}$ 
Surgical techniques commonly used to form the tracheal portal include a vertical incision or fenestration of the anterior trachea. These stomas can be fashioned quickly and close soon after extubation. ${ }^{[8]}$ However, recannulation can be difficult, and the risk of tracheal trauma is increased. ${ }^{[9]}$

The Björk flap is an anterior inverted U-shaped flap raised between the $2^{\text {nd }}$ and $4^{\text {th }}$ tracheal rings ${ }^{[8]}$ The tract that is formed is well-defined because the flap is inverted and sutured to the lower skin edge. This fixation of the trachea can cause dysphagia, but reduces the risk of displacement and usually facilitates replacement. ${ }^{[10]}$ However, it is important to be aware that if the tracheostomy tube is displaced the Björk flap can move, obstruct the trachea and prevent orotracheal intubation. ${ }^{[9]}$

Sutures can be placed through the tracheal wall either side of a surgically formed tracheostomy to hasten the formation of a mature stoma and may reduce the risk of tracheostomy tube displacement. These are called "stay sutures" and in one series, of the 104 patients who had a surgical tracheostomy formed with stay sutures none displaced while the tracheostomy tube displaced from 3 of the 101 patients who did not have stay sutures. ${ }^{[11]}$ Stay sutures may be lifesaving; these three patients died because of this complication. ${ }^{[1]}$

In the event of tracheostomy tube displacement upward and lateral traction on these sutures pulls the tracheal stoma closer to the skin surface of the tracheostomy tract which can greatly facilitate reinsertion of the displaced tube. ${ }^{[2,12,13]}$ However, stay sutures weaken the anterior trachea and can cut through the tracheal wall. Furthermore, if crossed or entangled, the tracheal stoma will be narrowed by traction on these stay sutures. This would exacerbate airway obstruction and prevent reinsertion of the tracheostomy tube. Therefore, stay sutures should be clearly marked "left" and "right." These stay sutures should also be taped to the skin of the neck and marked "Do Not Remove." These stay sutures can be removed after the first tracheostomy tube change.

The tracheal stoma and tracheostomy tract is often much smaller in patients who have had a percutaneous tracheostomy. In the event of accidental decannulation within 2 weeks, the airway should be secured by orotracheal intubation. Reinsertion of the tracheostomy tube will require either digital or instrumental redilation of the tract. This has a high risk of compromising the airway and should only be attempted by those experienced in performing a percutaneous tracheotomy in the presence of an anesthetist prepared for orotracheal intubation. When the tract has fully matured, direct repositioning may be possible.

\section{Formulating a Management Plan for the Patient With a Displaced Tracheostomy Tube}

If the upper respiratory tract is obstructed or the patient has had a laryngectomy oxygenation is not possible through any other route. While oxygen may be delivered via application of a facemask, trachymask or supraglottic airway over the stoma, this is an only temporizing measure, and the tracheostomy should be recannulated quickly. If a tracheostomy tube is displaced from an old well-formed tracheal stoma and tracheostomy tract, it may be possible to see into the trachea easily. In these cases, the associated morbidity and mortality are very low, and reinsertion of a tracheostomy tube via the tract is usually straightforward. A trial decannulation may be considered in this latter cohort.

Tracheostomy tube displacement within $72 \mathrm{~h}$ of formation is very challenging because the tract is not well established and may be lost if the tube is removed. ${ }^{[3,14]}$ If a tracheostomy tube is displaced within $72 \mathrm{~h}$ of formation and if the upper respiratory tract is patent, it is recommended that the airway should be secured by orotracheal intubation before replacement of the tracheostomy tube is attempted. Furthermore, replacement of the tracheostomy tube should only be attempted by an individual experienced at performing a tracheotomy. The tracheostomy matures over 10-14 days and tube displacement after this time may be easier to manage. ${ }^{[3,10]}$

So if the upper respiratory tract is patent, supply oxygen via a facemask and prepare for orotracheal intubation. If oxygenation can be maintained and the patient is not compromised, the tracheostomy tube should be replaced as soon as possible to preserve the tract. ${ }^{[12]}$

If the tract is not well defined or the tracheostomy cannot be recannulated easily, it is always safest to re-secure the airway by orotracheal intubation. However, this will usually require general anesthesia and paralysis with muscle relaxants. Furthermore, orotracheal intubation can be very difficult after tracheostomy. Hence, particularly, if the patient was being ventilated via the tracheostomy until it displaced, fiberoptic inspection of the upper respiratory tract and trachea should be considered. ${ }^{[15]}$ An experienced airway specialist should also be present and prepared for rapid sequence induction of anesthesia, and orotracheal intubation should difficulty arise.

The morbidity and mortality associated with tracheostomy tube displacement from a recently formed tracheal stoma in a patient without a patent upper respiratory tract are very high. In these difficult cases, recannulation may be facilitated by a guide passed via the stoma or a partially displaced tracheostomy tube. The optimum choice of guide is determined by the quality of the pretracheal tissues and the patency of the tract. The softer guides (e.g., suction catheter or nasogastric tube) may be too flexible to negotiate a relatively new tract that has collapsed into the pretracheal tissues. However, stiff guides such as a bougie or airway exchange catheter may damage friable and or infected, pretracheal tissue and create a false passage if advanced blindly.

It is therefore recommended that an Aintree airway exchange catheter (Cook Medical, USA) over a fibreoptic bronchoscope (FOB) is used for recannulation of a tracheal stoma with a 
Rajendram, et al.: Tracheostomy tube displacement

poorly formed tracheostomy tract, particularly in patients with a significant amount of pretracheal fat (i.e., obese patients). ${ }^{[16]}$ This advanced technique, which is described in more detail below, allows the placement of a guide for tracheostomy tube insertion into the trachea under direct vision. The fiber-optic bronchoscope can then be used to immediately confirm that the tracheostomy tube has been correctly reinserted into the trachea.

\section{Beware the Patient with a Fenestrated TRACHEOSTOMY}

It is important to know if the patient's tracheostomy tube has a fenestration. Any exchange guide passed blindly through a partially displaced fenestrated tracheostomy can catch on or slip through the fenestration. ${ }^{[17]}$ Removal or adjustment of the tracheostomy tube may be difficult if this occurs. The cause of these difficulties will not be immediately apparent as it will not be possible to see that the exchange guide has passed through the fenestration. Therefore if a fenestrated tracheostomy becomes displaced and use of a guide is required to facilitate replacement then either a nonfenestrated inner canula should first be inserted, or a FOB should be used to bypass the fenestration under direct vision.

\section{Cuffed and Uncuffed Tracheostomy Tubes}

Tracheostomy tubes can be cuffed or uncuffed. Tracheostomy tube cuffs can be filled with air, foam or water. The cuffs are designed to seal the airway to allow mechanical ventilation and reduce the risk of displacement.

It is important to note that air may diffuse through a cuff designed to be inflated with water. This may result in gradual deflation of the cuff which increases the risk of displacement. Before insertion of a tracheostomy tube with a foam-filled cuff, the air in the cuff must be removed using a syringe attached to the pilot port. Once the tube is in place, the syringe is removed to allow the cuff to reinflate spontaneously. Remember that the pilot tube remains open and so if the port is detached the foam-filled cuff will remain inflated. ${ }^{[6]}$ Water or air-filled cuffs deflate if the pilot port is detached. It is important to recognize this to avoid damaging the trachea and pretracheal tissue by attempting to remove a partially displaced tracheostomy tube with a fully inflated foam-filled cuff.

An inflated tracheostomy tube cuff impairs swallowing and the natural cough. Deflation of the cuff allows speech and improves laryngeal movement during swallowing but greatly increases the risk of displacement. A patient with a tracheal tube with an inflated cuff should not be able to speak. So if a tracheostomized patient can talk despite having an inflated cuff suspect that the tracheostomy is displaced.

Deflation of the cuff or use of an uncuffed tracheostomy tube is usually only considered for stable patients with well-formed tracts who are in the latter stages of weaning ventilatory support. In these patients, accidental decannulation is unlikely to have significant consequences and if the tube is displaced a trial decannulation should be considered if the patient is stable.

Most ventilators can compensate for a small leak around a tracheal tube. However, if a patient with an uncuffed tracheostomy deteriorates and requires significant mechanical ventilatory support, the tube should be replaced with a cuffed tube.

\section{Prevention of Tracheostomy Tube Displacement}

Sedation, suturing the flanges of the tracheostomy to the skin, specialized tracheostomy dressings, ties around the neck and connection of the tube to the ventilator with flexible tubing may reduce the risk of displacement. ${ }^{[3]}$ Regardless, healthcare professionals caring for patients with tracheostomy tubes must be prepared to manage airway emergencies in these patients at any time.

\section{Equipment that Should Be at the Bedside of Patients With a Tracheostomy}

Whether the patient is in the ICU or on a ward all the equipment required to execute emergency airway management plans must be kept immediately accessible and checked regularly. The nursing staff caring for the patient must be able to locate and correctly identify each item of this equipment swiftly. This is most readily facilitated by keeping some essential items at the patient's bedside in a "tracheostomy kit" with more specialist equipment kept on an airway management cart or resuscitation trolley.

Tracheostomy sets of the same type as the one the patient has in situ, in a range of sizes should be at the bedside. ${ }^{[12]}$ A source of oxygen, a suction device, and suction catheters should also be at the bedside. A resuscitation bag with facemask, a selection of endotracheal tubes (including one a half size smaller than the outer diameter of the tracheostomy tube) and capnography should also be immediately available but may be kept on a resuscitation trolley nearby. ${ }^{[4]}$ It is recommended that an Aintree intubation catheter (AIC) and a FOB are added to this list. These latter two devices are particularly useful in patients with a recently formed stoma in whom recannulation or orotracheal intubation is expected to be difficult. ${ }^{[16]}$

As important as making the equipment available is ensuring that all staff who could be called upon to attend the patient in an emergency are experienced in the use of this equipment and have the requisite skills to execute the predefined airway management plan. While the mantra "keep it simple and standardized" should be kept in mind, this is not always possible in patients with a tracheostomy. This cohort of patients may have very difficult airways, and complex, advanced strategies such as the combination of an AIC with a flexible bronchoscope may be required in an emergency. 
Rajendram, et al.: Tracheostomy tube displacement

\section{The Use of an Aintree Intubation Catheter With a Flexible Bronchoscope}

The AIC is a flexible, disposable, hollow ventilation/exchange catheter with a length of $56 \mathrm{~cm}$ and an internal diameter of $4.8 \mathrm{~mm}$. The AIC can be mounted on a FOB with an outer diameter up to $4 \mathrm{~mm}$ and can be used to facilitate orotracheal intubation through a laryngeal mask airway or insertion of tracheostomy tubes with an internal diameter of at least $7.0 \mathrm{~mm} \cdot{ }^{[17,18]}$ The AIC and bronchoscope need to be well lubricated before the procedure.

The use of this technique to replace tracheostomy tubes has several potential advantages. ${ }^{[16,19]}$ The directional control of the FOB assists passage into the trachea, and correct placement within the trachea can be confirmed immediately. The risks of forming a false passage in the pretracheal space or losing the airway are thereby vastly reduced.

This technique is particularly useful if sedation and paralysis with muscle relaxants are undesirable, for example during weaning from ventilatory support. The only minimal increase in sedation is required, and spontaneous ventilation can be maintained throughout. However, it is useful to note that the AIC is supplied with a removable $15 \mathrm{~mm}$ Rapi-fit ${ }^{\mathbb{B}}$ connector (William Cook Europe, Denmark) that allows oxygen insufflation via the AIC if required. ${ }^{[17]}$

This technique for tracheostomy tube replacement requires advanced airway skills and so should only be considered by clinicians with significant experience in the management of tracheostomies who are also familiar with the manipulation of an AIC mounted on a FOB ${ }^{[16]}$ Experience can be gained on partial task trainers for the insertion of tracheostomy tubes and by using this technique for routine tracheostomy tube changes.

The AIC is particularly suitable for use with this technique. Other hollow exchange catheters such as the Cook airway exchange catheter are much more rigid than the AIC, and more difficult to negotiate through the curve of rigid tracheostomy tubes. ${ }^{[20]}$ This distinction is less relevant for more flexible tracheostomy tubes.

\section{Conclusion}

With an incidence under $1 / 1000$ tracheostomy tube days ${ }^{[1]}$ tracheostomy tube displacement is very uncommon. However, the associated mortality is very high. While a decannulation trial may be appropriate in stable patients with well-formed tracheostomy tracts, rapid repositioning or replacement is usually required. Multiple attempts to blindly reposition the tube may disrupt the preexisting tract and cause significant hemorrhage and or surgical emphysema. The safest approach is always to secure the airway under direct vision either via laryngoscopy and orotracheal intubation IF the upper airway is patent. If the upper airway is NOT patent, there is no alternative but to re-secure the airway via the trachea. In this situation, the use of a FOB to guide and confirm replacement of the tracheostomy should strongly be considered. However, even experienced airway specialists may have difficulty obtaining the information and equipment required to formulate and execute an effective management plan in the chaos which ensues when a critically ill patient's tracheostomy tube is displaced. It is therefore strongly recommended that relevant information about a patient with a tracheostomy tube in situ is made visible at their bedside. ${ }^{[5,6]}$ This extremely vulnerable cohort of patients also requires specific, individualized, written plans for the management of tracheostomy-related emergencies. All the equipment required to execute these plans should be kept immediately available. The nursing staff and doctors should be familiar with this equipment and trained in its use.

\section{Financial support and sponsorship}

Nil.

\section{Conflicts of interest}

There are no conflicts of interest.

\section{References}

1. Kapadia FN, Tekawade PC, Nath SS, Pachpute SS, Saverkar SS, Bhise RA, et al. A prolonged observational study of tracheal tube displacements: Benchmarking an incidence $<0.5-1 \%$ in a medical-surgical adult Intensive Care Unit. Indian J Crit Care Med 2014;18:273-7.

2. Cook T, Woodall N, Frerk C. Major Complications of Airway Management in the United Kingdom. National Audit Project 4. The Royal College of Anaesthetists and the Difficult Airway Society; March, 2011.

3. Mirza S, Cameron DS. The tracheostomy tube change: A review of techniques. Hosp Med 2001;62:158-63.

4. Bernard AC, Kenady DE. Conventional surgical tracheostomy as the preferred method of airway management. J Oral Maxillofac Surg 1999;57:310-5.

5. McGrath BA, Bates L, Atkinson D, Moore JA; National Tracheostomy Safety Project. Multidisciplinary guidelines for the management of tracheostomy and laryngectomy airway emergencies. Anaesthesia 2012;67:1025-41.

6. Rajendram R, Barker G. Self-expanding foam-filled tracheostomy tube cuffs and the management of airway emergencies. Anaesthesia 2013;68:436-7.

7. Chew JY, Cantrell RW. Tracheostomy. Complications and their management. Arch Otolaryngol 1972;96:538-45.

8. Bjork VO. Partial resection of the only remaining lung with the aid of respirator treatment. J Thorac Cardiovasc Surg 1960;39:179-88.

9. Crawley BE. Technique of tracheostomy. In: Feldman SA, Crawley BE, editors. Tracheostomy and Artificial Ventilation in the Treatment of Respiratory Failure. $3^{\text {rd }}$ ed. London: Edward Arnold; 1977. p. 53-63.

10. Young JS, Brady WJ, Kesser B, Mullins D. A novel method for replacement of the dislodged tracheostomy tube: The nasogastric tube "guidewire" technique. J Emerg Med 1996;14:205-8.

11. Lee SH, Kim KH, Woo SH. The usefulness of the stay suture technique in tracheostomy. Laryngoscope 2015;125:1356-9.

12. Seay SJ, Gay SL, Strauss M. Tracheostomy emergencies. Am J Nurs 2002;102:59, 61, 63.

13. Burke A. The advantages of stay sutures with tracheostomy. Ann R Coll Surg Engl 1981;63:426-8.

14. Quigley RL. Tracheostomy - An overview. Management and complications. Br J Clin Pract 1988;42:430-4.

15. Vyvyan HA. Complications of dislodged tracheostomy. Anaesthesia 1994;49:558-9. 
Rajendram, et al.: Tracheostomy tube displacement

16. Rajendram R, McGuire N. Repositioning a displaced tracheostomy tube with an Aintree intubation catheter mounted on a fibre-optic bronchoscope. Br J Anaesth 2006;97:576-9.

17. Atherton DP, O'Sullivan E, Lowe D, Charters P. A ventilation-exchange bougie for fibreoptic intubations with the laryngeal mask airway. Anaesthesia 1996;51:1123-6.
18. Zura A, Doyle DJ, Orlandi M. Use of the Aintree intubation catheter in a patient with an unexpected difficult airway. Can J Anaesth 2005;52:646-9.

19. McNamara J, Chisholm DG. Use of a fibreoptic intubating laryngoscope to replace a misplaced tracheostomy tube. Anaesthesia 1996;51:894.

20. Bhuvanagiri A, Thirugnanam M, Rehman K, Grew NR. Repositioning a displaced tracheostomy tube. Br J Anaesth 2007;98:276. 